\title{
Childhood Neurocysticercosis: Clinico-Radiological Profile and Outcome
}

\author{
Shrestha BM 1 . \\ 1. Dr. Binod Man Shrestha Consultant Paediatrician, Lumbini Zonal Hospital \\ Address for correspondence: nepas@ healthnet.org.np, Lumbini Zonal Hospital
}

\begin{abstract}
Neurocysticercosis (NCC) is a major cause of neurological illness worldwide. It is the most common identifiable cause of partial seizure especially in the children of developing world. There is insufficient information about NCC in children in Nepal. This study was, therefore, conducted to evaluate the clinical, neuro-radiographic and therapeutic aspects of NCC in children. Material and Methods: 68 children with this neurocysticercosis were studied prospectively in 20 months in the Lumbini Zonal Hospital (LZH), a secondary-level-referral hospital in the Western Nepal. The diagnosis of NCC was based primarily on the neuro-imaging (CT/MRI) findings. Results: The patients were predominantly females (nearly 65\%) with age ranging from 2 to 14 years. Preschool-age children constituted $10 \%$ of the patients. The three common manifestations were seizures $(91 \%)$, headache and or vomiting $(38 \%)$ and hemi and or monoparesis (15\%). CT/MRI demonstrated a single parenchymal ring or nodular enhancing lesion (REL) in $84 \%$ of cases with perilesional oedema in nearly $90 \%$ of cases. A large majority of patients were treated only with the anticonvulsant drugs (ACDs) for 9 months. Follow-up with repeat CT after 6 months showed a complete resolution of NCC in most of the cases without the need for cysticidal treatment. Conclusion: NCC should be considered first in the differential diagnosis of new-onset seizure among the children of developing countries, where taeniasis is endemic. Most of the patients with NC do not need anticysticercal therapy.
\end{abstract}

Keywords: Seizure, Children, Cysticercosis, CNS, Western Nepal.

\section{Introduction:}

Neurocysticercosis (NCC) is the most common parasitic disease of the central nervous system (CNS) in the world ${ }^{1}$, and is highly endemic in the Asian developing countries like Nepal. NCC is caused by the tissue-invading larvae (Cysticercus Cellulosae) of the pork-tapeworm; Taenia Solium. Humans acquire tapeworm infection via the faecooral route, usually by the ingestion of food or water contaminated with the eggs of T. solium ${ }^{2}$. The disease may, therefore develop even in individuals who do not eat pork. NCC relatively more common in certain rural communities due to poor sanitation, use of human faeces for fertilizing plants in gardens or farms, and lack of controlled pens for pigs ${ }^{3}$.

Neurological symptoms arise as a result of a host's inflammatory response to the dying parasite ${ }^{3}$. The most characteristic feature of NCC in children is the acute onset of partial seizure, which may occur in more than $80 \%$ of cases ${ }^{4}$. Other frequent manifestations are intracranial hypertension, learning disability, behavioral changes and psychiatric manifestations $\mathbf{1 , 5 , 6}$. More than $80 \%$ of children with NCC have normal physical and neurological examination ${ }^{3,5}$. Besides Computerized Tomography Scan (CT) and Magnetic Resonance Imaging (MRI), serological tests such as enzymelinked immuno-transfer blot assay (EITB), enzyme-linked immunosorbent assay (ELISA) are also important modalities for the diagnosis of NCC. EITB has a sensitivity of $72 \%$ and a specificity of $96 \%$ in children with just one lesion ${ }^{7}$. High levels of TNF-alpha have been shown in the cerebro-spinal fluid (CSF) from children with active $\mathrm{NC}^{8}$. Stool examination of these patients may show the presence of ova or proglottids of the tapeworms in $25-40 \%$ of cases ${ }^{2,9}$. The diagnostic criteria of NCC laid down by Rosenfeld, et al ${ }^{\mathbf{1 0}}$ are:

(i) Surgical biopsy findings,

(ii) Radiographic findings consistent with NCC as well as diagnostic serum and or cerebrospinal fluid titers, or

(iii) Consistent radiographic findings and a compatible epidemiological history.

Antiepileptic Drugs (AEDs) are of universal benefit to stop seizure. Use of cysticidal medication is considered to be controversial $^{3}$. Recent data suggest that a one-week therapy with albendazole, is as effective as a four week therapy in children with NCC having one to three lesions ${ }^{11}$. The longterm prognosis for treated patients with NCC is excellent ${ }^{3,10}$.

\section{Materials and Methods:}

Ninty-three (93) consecutive children presenting in the emergency and pediatric outpatient departments of Lumbini Zonal Hospital (LZH) with new-onset seizure or unexplained sudden loss of consciousness (LOS) between 2059-01-01 and 2060-08-29 (equivalent to 20 months of the local calendar) were selected for the study. But only 68 patients with CT/ MRI findings consistent with NCC were included in the study. Total and differential leucocyte count, erythrocyte sedimentation rate (ESR) and Mantoux tests were done in all of these patients. Excluded from the study were those patients whose CT/MRI findings were either not consistent with NCC (6 patients) or whose CT or MRI reports were not available at all (19 patients). All of these 68 patients were treated initially with the anti epileptic drug; Carbamazepine (CBZ). Five of 
them also received Dexamethasone plus Albendazole. Patients on CBZ were followed up initially for a month to find out the side effects. Six months after starting AEDs, CT or MRIs were repeated. AEDs were slowly weaned in 3 months after the complete resolution of the lesion.

\section{Results:}

Age incidence: Age of the patients ranged from 2 to 14 years (mean-5.8 years), with peak incidence among 8 to 10 years children. Preschool-age children $(<6$ years $)$ constituted only seven $(10.3 \%)$ children. Forty-seven $(69.1 \%)$ of the patients were older than 7 years.

Table 1: Showing the age incidence.

\begin{tabular}{|l|c|c|}
\hline Age (years) & No. of patients & Percentage \\
\hline$<2$ & 0 & 0 \\
\hline $2-4$ & 5 & 7.4 \\
\hline $5-7$ & 16 & 23.5 \\
\hline $8-10$ & 26 & 38.2 \\
\hline$>10$ & 21 & 30.9 \\
\hline Total & 68 & 100 \\
\hline
\end{tabular}

Sex incidence: Female patients constituted nearly $65 \%$ $(n=43)$ of the total cases.

Table 2: Showing sex incidence.

\begin{tabular}{|l|c|c|}
\hline Sex & No of cases & Percentage \\
\hline Male & 25 & 35.3 \\
\hline Female & 43 & 64.7 \\
\hline Total & 68 & 100 \\
\hline
\end{tabular}

Clinical presentation: The most frequent manifestation was seizure in $62(91 \%)$ patients, followed by headache and or vomiting in $26(38 \%)$ patients and neurological deficits like; hemi and or monoparesis in $10(15 \%)$ patients. Partial seizures were present in $85 \%$ of cases. Complex partial seizure $(48.5 \%)$ predominated over simple partial seizure $(29.4 \%)$. One of the patients had only sensory disturbances in his left upper limb (? Simple partial seizures)

Table 3: Showing the clinical features.

\begin{tabular}{|l|c|c|}
\hline Clinical features & No. of cases & Percentage \\
\hline Seizure & 62 & 91.2 \\
\hline Headache/vomiting & 26 & 38.2 \\
\hline Hemi/monoparesis & 10 & 14.7 \\
\hline Unexplained sudden LOC & 6 & 8.8 \\
\hline Fever & 5 & 7.4 \\
\hline
\end{tabular}

Laboratory studies: Neutrophilia and eosinophilia were present in $30 \%$ and $19 \%$ of cases respectively. High ESR > 20 $\mathrm{mm} / \mathrm{hr}$ were found only in $19 \%$ of cases. Mantoux tests of all patients with neuro-imaging findings consistent with NCC were negative. ELISA for NCC done elsewhere was positive in four cases.

Neuro-imaging (CT/MRI) studies: Single parenchymal ring/nodular enhancing lesion/s (REL) were found in 57
(83.8\%) patients. Multiple RELs were seen in only 7 (10.3\%) cases. Similarly calcifications were seen in only $4(5.9 \%)$ cases. Perilesional oedema was noticed in $61(89.7 \%)$ cases.

Treatment \& outcome: Seven out of ninety-three (7.5\%) patients treated with CBZ developed rashes, while 1/93 patient developed leucopenia. CBZ was, therefore replaced by clobazam in 4 patients, and phenytoin in another 4 patients. One of the patients on phenytoin developed vertigo $\&$ ataxia after she took a high dose $(300 \mathrm{mg})$ of the drug by mistake. Correction of the dosage improved her completely. Additional therapy with albendazole plus dexamethasone was given to 5 patients. One patient developed severe headache. Discontinuation of albendazole ultimately relieved her problem. Complete resolution of the lesion/s in CTs repeated after 6 months was seen in a large majority of the patients. Recurrence of seizure 9 months after the discontinuation of AED occurred in 2 cases. No patients died nor suffered from any major complications in this study.

\section{Discussion}

This study showed that there were more girls suffering from NCC as compared to boys (65\% vs 35\%). This finding is similar to those of Ruiz-Garcia, et al ${ }^{\mathbf{1}}$, and Thakur, et al ${ }^{\mathbf{1 2}}$, but in contrast to the finding of Morales et al, who found a male preponderance ${ }^{5}$. In fact no sexual predilection exists ${ }^{3}$. Current study showed a $10 \%$ incidence of NCC in preschoolage children. Nearly $70 \%$ of children were older than 7 years. There were no patients younger than 2 years. These findings are similar to those reported by Kossoff and Thakur et al ${ }^{3,12}$. Lower incidence of NCC in the preschool-age children is because of the prolonged incubation period (as long as 5 years) of T. solium as well as the nutritional habits young children's 3,13

$91 \%$ of children in this study presented with seizures, mostly focal in nature (85\%). This finding is similar to those reported by Singhi et al and Kalra et al ${ }^{6,14}$. Solitary parenchymal lesions as in our study mostly produce focal seizures and focal neurological deficit ${ }^{2}$. Headache and vomiting, which may be the features of raised intracranial pressure, were present in $38 \%$ of cases in the current study. Similar presentations were seen in $30 \%$ and $44 \%$ of cases in other two studies ${ }^{6,14}$. Present study showed a nearly $15 \%$ incidence of focal neurological deficit (hemi/monoparesis), which was observed in only $4 \%$ of cases in a study done by Singhi et al $^{6}$. All except one patient with this deficit improved completely within 24 hours (Todd's palsy). Hemi/monoparesis results either from the cyst or from Todd paralysis after a partial seizure $^{3}$. Fever occurred in $7.4 \%$ of cases in the present study, mostly because of the associated pneumonia. However Rosenfeld et al found fever in none of the children with NCC in their study ${ }^{10}$.

Blood examinations in this study revealed neutrophilia and eosinophilia in nearly $30 \%$ and $20 \%$ of cases respectively with high ESR in $20 \%$ of cases. But these laboratory studies are neither sensitive for nor predictive of the diagnosis of NCC ${ }^{10}$. Imaging studies showed single parenchymal RELs in $84 \%$ of cases with perilesional oedema in $90 \%$ of cases. Singhi and Talukdar et al in their studies reported similar CT findings ${ }^{6,15}$. 
Three patients presenting with complex partial seizures had normal CT reports, and were excluded from the study. But $10-20 \%$ of the children with NCC may have normal CT/MRI reports ${ }^{3}$. Multiple RELs/cysts, which were seen in $10 \%$ of patients in the current study, were found in $7 \%$ of cases in another study ${ }^{6}$. Six percent of patients had single or multiple calcifications, which are observed less frequently in children as compared to adults ${ }^{3}$. The natural history of cysts is to resolve by complete resorption or calcification ${ }^{2}$.

CBZ had to be discontinued in 8 out of 93 patients because of the appearances of rashes in 7 patients and leucopenia in 1 patient. Rash and leucopenia are the important side effects of CBZ, for which close follow-up of patients is needed at least for the initial 1-3 months. One of the patients getting albendazole developed severe headache within 24 hours. This headache was probably because she had a large perilesional oedema. Destruction of cysts by antihelminthic drug if given to a child with severe oedema, can cause further inflammation and swelling resulting into headache, vomiting and seizure ${ }^{3}$. Prophylactic treatment with dexamethasone is beneficial in decreasing the severity of such neurological symptoms ${ }^{3}$.

CT repeated after six months of AED therapy showed a complete resolution of the cystic lesion/s in a great majority of cases in the present study. Spontaneous resolution of NCC without the need for cysticidal treatment has been reported before also, by Morales et al ${ }^{5}$. In fact most lesions of NCC resolve spontaneously within 2-3 months ${ }^{3}$. Although none of the patients in this study died, deaths have been reported in the earlier study because of chronic arachnoiditis ${ }^{1}$.

\section{Conclusion \& Recommendation}

Data suggest that partial seizures and single parenchymal lesions are the most common clinical and neuroradiographic manifestations of NCC in children. It is recommended to consider NCC as a differential diagnosis of new-onset partial seizure in a Nepalese child. Albendazole should be used in case of multiple cystic lesions with no or minimal perilesional oedema. Most solitary lesions resolve completely and spontaneously without the need for antiparasitic medication.

\section{Acknowledgement}

I would like to thank our medical superintendent, Dr Nand Pd Sharma for allowing me to do this study. I also want to thank Dr Sushma Lamichhane and Mr. Meen B. Somai for their help.

\section{References}

1. Ruiz-Garcia M, Gonzalez-Astiazaran A, Rueda-Franco F et al. Neurocysticercosis in Children : Clinical Experience in 122 Patients. Child Nervous System. 1997; 13:608-12.

2. Blanton R. Cysticercosis. In Behrman RE, Kliegman RM, Jenson HB(editors) Nelson Textbook of Pediatrics. $17^{\text {th }}$ Ed Philadelphia, Saunders Company, 2004, pp.117172.

3. Kossoff EH. Neurocysticercosis. March 2003. (available at http://www.emedicine.com/Ped/topic 1573.htm).

4. Vazquez V, Sotelo J. The Course of Seizures After Treatment for Cerebral Cysticercosis. N Engl J med. 1992; 327:696-701(Medline).

5. Morales NM, Agapejev S, Morales RR, et al. Clinical Aspects of Neurocysticercosis in Children. Pediatric Neurology. 2000; 22:287-91.

6. Singhi P, Ray M, Singhi S, et al. Clinical Spectrum Of 500 Children With Neurocysticercosis And Response Too Albendazole Therapy. J Child Neurol. 2000;15:207-13.

7. Aguilar-Rebolledo F, Meza-Lucas A, Torres J, et al. Evaluation of the Enzyme-Linked Immunoelectrotransfer Blot Assay (EITB) for Diagnosis of Neurocystocercosis in Children. J Child Neurol.2002; 17:416-20.

8. Aguilar-Rebolledo F, Cedillo-Rivera-R, LlagunoViolante P, et al. Interleukin Levels in Cerebrospinal Fluid from Children with Neurocysticercosis. American J Trop Med \& Hyg. 2001; 64:35-40.

9. Ferreira MS, Costa-Cruz JM, Nishioka SA, et al. Neurocysticercosis in Brazilian Children: Report of 10 Cases. Trop Med \& parasit. 1994;45:49-50.

10. Rosenfeld EA, Byrd SE, Shulman ST. Neurocysticercosis among Children in Chicago. Clin Infect Dis. 1996; 23:262-8.

11. Shinghi P,Dayal D, Khandelwal N. One Week Versus Four Weeks of Albendazole Therapy for Neurocysticercosis in Children; A Randomized, PlaceboControlled Double Blind Trial. Ped Infect Dis J . 2003;22:268-72.

12. Thakur LC, Anand KS. Childhood Neurocyrticercosis in South India. Ind J Ped. 1991;58:815-9.

13. Vasconcelos M M, Ramos MR, Schwan PJ, .et al. Neurocysticercosis in a Preschool-Age Child; Case Report . Arquivos de Neuro-Psiquiatria.2000; 58:90212.

14. Kalra V, Sethi A. Childhood NeurocysticercosisEpidemiology, Diagnosis and Course. Acta Paediatrica Japonica.1992; 37:365-70.

15. Talukdar B, Saxena A, popli VK et al. Neurocystcercosis in Children: Clinical Characteristics and Outcome. Annals of Tropical Paediatrics. 2002; 22:333-9. 